Louisiana State University

LSU Digital Commons

Faculty Publications

Department of Physics \& Astronomy

$12-7-2010$

\title{
Risk of second malignant neoplasm following proton versus intensity-modulated photon radiotherapies for hepatocellular carcinoma
}

\author{
Phillip J. Taddei \\ University of Texas MD Anderson Cancer Center \\ Rebecca M. Howell \\ University of Texas MD Anderson Cancer Center \\ Sunil Krishnan \\ University of Texas MD Anderson Cancer Center \\ Sarah B. Scarboro \\ University of Texas MD Anderson Cancer Center \\ Dragan Mirkovic \\ University of Texas MD Anderson Cancer Center
}

See next page for additional authors

Follow this and additional works at: https://digitalcommons.Isu.edu/physics_astronomy_pubs

\section{Recommended Citation}

Taddei, P., Howell, R., Krishnan, S., Scarboro, S., Mirkovic, D., \& Newhauser, W. (2010). Risk of second malignant neoplasm following proton versus intensity-modulated photon radiotherapies for hepatocellular carcinoma. Physics in Medicine and Biology, 55 (23), 7055-7065. https://doi.org/10.1088/0031-9155/55/ $23 / 507$

This Article is brought to you for free and open access by the Department of Physics \& Astronomy at LSU Digital Commons. It has been accepted for inclusion in Faculty Publications by an authorized administrator of LSU Digital Commons. For more information, please contact ir@lsu.edu. 


\section{Authors}

Phillip J. Taddei, Rebecca M. Howell, Sunil Krishnan, Sarah B. Scarboro, Dragan Mirkovic, and Wayne D. Newhauser 


\title{
Risk of second malignant neoplasm following proton versus intensity-modulated photon radiotherapies for hepatocellular carcinoma
}

\author{
Phillip J Taddei ${ }^{1}$, Rebecca M Howell, Sunil Krishnan, Sarah B Scarboro, Dragan Mirkovic, \\ and Wayne D Newhauser \\ Division of Radiation Oncology, Unit 1202, The University of Texas M D Anderson Cancer \\ Center, 1515 Holcombe Blvd, Houston, TX 77030, USA
}

\begin{abstract}
Hepatocellular carcinoma (HCC), the sixth most common cancer in the world, is a global health concern. Radiotherapy for HCC is uncommon, largely because of the likelihood of radiationinduced liver disease, an acute side effect that is often fatal. Proton beam therapy (PBT) and intensity-modulated radiation therapy (IMRT) may offer HCC patients a better option for treating the diseased liver tissue while largely sparing the surrounding tissues, especially the non-tumor liver. However, even advanced radiotherapies carry a risk of late effects, including second malignant neoplasms (SMNs). It is unclear whether PBT or IMRT confers less risk of an SMN than the other. The purpose of this study was to compare the predicted risk of developing an SMN for a patient with HCC between PBT and IMRT. For both treatments, radiation doses in organs and tissues from primary radiation were determined using a treatment planning system; doses in organs and tissues from stray radiation from PBT were determined using Monte Carlo simulations and from IMRT using thermoluminescent dosimeter measurements. Risk models of SMN incidence were taken from the literature. The predicted absolute lifetime attributable risks of SMN incidence were $11.4 \%$ after PBT and $19.2 \%$ after IMRT. The results of this study suggest that using proton beams instead of photon beams for radiotherapy may reduce the risk of SMN incidence for some HCC patients.
\end{abstract}

\section{Introduction}

\begin{abstract}
Worldwide, liver cancer is the sixth most common cancer and ranks third as the cause of cancer-related deaths, with an estimated 711000 new cases diagnosed and approximately 680000 cancer-related deaths in 2007. Liver cancer mainly occurs in China and other developing countries (Garcia et al 2007). It is less common in the United States than elsewhere: in 2009, US incidence was predicted at 22620 cases and mortality at 18160 (Horner et al 2008). Hepatocellular carcinoma (HCC) accounts for 70-85\% of liver cancer cases worldwide (Perz et al 2006). Within the US, the incidence of HCC per hundred thousand people has tripled between 1975 and 2005, from 1.6 cases to 4.9, largely because of a rapid increase in the number of people infected with the hepatitis $\mathrm{C}$ virus. Although the one-year survival rate of US patients with $\mathrm{HCC}$ has improved in the last decade, it is still below 50\%, and there is considerable room for improvement (Altekruse et al 2009).
\end{abstract}

\footnotetext{
(C) 2010 Institute of Physics and Engineering in Medicine

${ }^{1}$ Author to whom any correspondence should be addressed.ptaddei@mdanderson.org.

(Some figures in this article are in colour only in the electronic version)
} 
Relative to other alternatives to surgery, radiotherapy historically has not been widely employed as a treatment option for HCC, largely because of the perceived risk of radiationinduced liver disease (RILD). RILD is an acute effect that occurs within 6 months of irradiation, and it is commonly lethal within a few months of diagnosis. With recent improvements in modeling the risk of RILD and in radiation planning and delivery techniques (such as the use of conformal, image-guided, respiratory-gated and chargedparticle therapies), it appears increasingly probable that curative doses of radiation can be administered to partial liver volumes while sparing the portion of the liver not involved by tumor (hereafter referred to as 'non-tumor liver') (Krishnan et al 2008). This option is particularly appealing as a bridge to transplantation in patients awaiting a donor liver.

In addition to RILD, survivors of HCC who undergo radiotherapy are at risk of radiation carcinogenesis, which is the late effect of greatest concern for radiotherapy patients with good prognoses for long-term survival. Minimizing the radiation dose to normal tissues throughout the body is important in avoiding second malignant neoplasms (SMNs). Normal tissue exposures may be minimized using intensity-modulated radiation therapy (IMRT) or proton beam therapy (PBT) (Zurlo et al 2000, Krishnan et al 2008, Dawson 2009). Both IMRT and PBT deliver the conformal dose to the clinical target volume (CTV), but comparative SMN risk assessment is challenging (cf Newhauser et al 2009), requiring knowledge of therapeutic, scatter and leakage radiation doses. Taddei et al (2009a) predicted the equivalent dose from stray radiation throughout the body of a patient receiving PBT for HCC using Monte Carlo simulations of the treatment; the doses from the primary proton fields were not reported. Howell et al (2010) described a methodology for quantifying the radiation dose from both primary and stray radiation in photon radiotherapy treatments, including IMRT. They applied this methodology to the same patient studied in this work, quantifying the absorbed dose from both primary and stray (i.e. scatter and leakage) radiation associated with IMRT. However, no previous reports directly compared the predicted risks for developing radiogenic SMNs following IMRT and PBT.

The aims of this study were to predict and compare the risks of SMN incidence after IMRT and PBT for a patient with primary HCC. Organ and tissue doses were determined throughout the body from primary and stray radiation. Based on these organ doses and risk models from the literature, we predicted the organ-specific risks of SMN incidence.

\section{Methods and materials}

\subsection{Treatment planning}

Two treatment plans were designed separately for a patient diagnosed with $\mathrm{HCC}$ at our clinic, one for PBT and one for IMRT, each according to the standard of care of our institution. The intent of the treatment was to cure the primary lesion. The patient was a 59year-old male, with a height of $174 \mathrm{~cm}$ and a mass of $93.9 \mathrm{~kg}$; he was randomly selected among HCC patients. Our study was performed under a protocol approved by our institutional review board for retrospective treatment planning studies. The treatment plans were based on the standard of care at our institution for PBT and IMRT treatments of liver cancer. Free-breathing kilovoltage computed tomography (CT) images were acquired for treatment planning purposes; the images extended from the patient's mid-thigh to the top of the head, and the slice thickness was $5 \mathrm{~mm}$. Organ and tissue structures were contoured and PBT and IMRT plans were created using a commercial TPS (Eclipse; Varian Medical Systems, Inc., Palo Alto, CA) and the free-breathing CT image set (Newhauser et al 2007a). The following tumor volumes were contoured: the gross tumor volume, the CTV (i.e. the gross tumor volume plus subclinical disease) and the planning target volume (PTV) (i.e. CTV plus internal and setup margins for the IMRT plan). 
The IMRT plan was optimized for nine 6-MV photon fields so that $95 \%$ of the prescribed dose of 60 Gy would be delivered to $100 \%$ of the PTV. The IMRT fields were shaped laterally with dynamic multi-leaf collimator settings, four of which were coplanar and five were non-coplanar. The normal tissue objectives included constraints of less than $28 \mathrm{~Gy}$ mean dose to the non-tumor liver, less than $40 \%$ of the non-tumor liver receiving $30 \mathrm{~Gy}$, less than $50 \%$ of the right (ipsilateral) kidney receiving 20 Gy and less than $30 \%$ of the left (contralateral) kidney receiving $20 \mathrm{~Gy}$. Details of the IMRT plan are reported in a companion article (Howell et al 2010).

The PBT plan comprised two proton fields to deliver a prescribed dose of $60 \mathrm{~Gy}$ to $100 \%$ of the CTV. To facilitate a direct comparison with the IMRT plan, the PBT plan was renormalized so that $95 \%$ of the PTV received $60 \mathrm{~Gy}$ as was the case for the IMRT plan. The two fields were in the posterior oblique orientation, with gantry angles of $220^{\circ}$ and $135^{\circ}$, couch angles of $45^{\circ}$ and $-40^{\circ}$, penetration ranges in the patient of $20.1 \mathrm{~cm}$ water equivalent thickness (WET) and $25.0 \mathrm{~cm}$ WET and spread-out Bragg peak widths of $14 \mathrm{~cm}$ WET and $13 \mathrm{~cm}$ WET for field 1 (PO1) and field 2 (PO2), respectively. Beam shaping devices for the PBT treatment unit included a range modulator wheel, scattering foil, range shifter, fielddefining collimator and range compensator; the latter two components accommodated collimated fields up to $18 \times 18 \mathrm{~cm}^{2}$ in size. Details of the PBT treatment plan were reported previously (Taddei et al 2009a).

For predicting risk of SMN incidence, we also contoured the whole liver, non-tumor liver, kidneys, stomach, colon, lungs, prostate, bladder, thyroid, red bone marrow, non-tumor body tissue and several other structures. The following two sections describe the equipment and methods used for determining the doses to these organs and tissues.

\subsection{Determining organ doses for PBT}

For PBT, the absorbed doses from primary radiation were calculated using the TPS, and the stray radiation doses were determined using a Monte Carlo system. Absorbed dose from the primary proton field in each voxel, $D_{v}$ (pri, PBT), was calculated in $2 \times 2 \times 2.5 \mathrm{~mm}^{3}$ voxels throughout the patient's body. $D_{T}$ (pri, PBT) was the mass-weighted average absorbed dose for all voxels within a particular organ, $T$, or

$$
D_{T}=\frac{\sum_{v \text { in } T} D_{v} \rho_{v}}{\sum_{v \text { in } T} \rho_{v}},
$$

where $\rho_{v}$ is the mass density of the tissue within a voxel. The equivalent dose, $H_{T}$, was the product of the mean radiation weighting factor, $\overline{w_{R}}$, and $D_{T}$, or

$$
H_{T}=\overline{w_{R}} D_{T},
$$

where $\overline{w_{R}}$ was taken as 1 for primary proton fields, as suggested by the National Council on Radiation Protection and Measurements (NCRP 1993) for incident protons with energy greater than $100 \mathrm{MeV}$.

To determine the doses from stray radiation, we used the Monte Carlo Proton Radiotherapy Treatment Planning (MCPRTP) system (Newhauser et al 2007a, 2008b), which uses the Monte Carlo N-Particle eXtended (MCNPX) code version 2.6b (Pelowitz 2008). In PBT, secondary neutrons are the chief source of the equivalent dose from stray radiation (Agosteo et al 1998, Fontenot et al 2008, Zheng et al 2008). Neutrons are produced both in the patient 
(analogous to patient scatter in photon radiotherapy) and in the treatment unit (analogous to leakage and collimator scatter in photon radiotherapy). The geometric model in the Monte Carlo simulations included all components of the treatment unit and detailed CT-based voxelized phantom representing the patient, in which the Hounsfield unit value for each $4 \times$ $4 \times 5 \mathrm{~mm}^{3}$ voxel was converted to a mass density and a material composition (Newhauser $e t$ al 2007b, 2008a, Taddei et al 2009b). In the MCPRTP system, $D_{v}$ was calculated for neutrons produced in the treatment unit (external neutrons), $D_{v}\left(n_{\text {ext }}\right)$, and for neutrons produced in the patient (internal neutrons), $D_{v}\left(n_{\text {int }}\right)$, in separate simulations for each treatment field. The corresponding mean absorbed doses, $D_{T}\left(n_{\text {ext }}\right)$ and $D_{T}\left(n_{\text {int }}\right)$, were calculated using equation (1) and the $H_{T}$ values were calculated using equation (2). The $\overline{w_{R}}$ values for external neutrons were determined for each treatment field based on the Monte Carlo simulations of the neutron spectral fluence incident upon the patient, following the recommendations of the International Commission on Radiological Protection (ICRP) Publication 92 (2003). The $\overline{w_{R}}$ values for internal neutrons were estimated based on the calculations of Zheng et al (2008). Finally, for each treatment field, the equivalent dose from stray neutron radiation, $H_{T}($ str, PBT), was the sum of the external and internal neutron contributions, or

$$
H_{T}(\operatorname{str}, \operatorname{PBT})=H_{T}\left(n_{\text {ext }}\right)+H_{T}\left(n_{\text {int }}\right) .
$$

These values were normalized following the methods reported by Taddei et al (2009c). Details of the methods of determining $H_{T}$ (str, PBT) were published previously (Taddei et al 2009a). They reported $\overline{w_{R}}$ values for external neutrons of 8.89 and 8.94 for fields PO1 and $\mathrm{PO}$, respectively, and $\overline{w_{R}}$ values for internal neutrons at 9.6 for each field.

\subsection{Determining organ doses for IMRT}

For IMRT, dose volume histogram (DVH) data were exported from the TPS and used to determine the absorbed dose from primary radiation to in-field organs. Thermoluminescent dosimeter (TLD) measurements were made in an anthropomorphic phantom to determine absorbed dose values from stray radiation for out-of-field organs. Either calculated doses or a combination of calculated and measured doses were used to determine absorbed dose for partially in-field organs. The methods to determine the organ doses for the IMRT plan were described, and the organ dose results were reported elsewhere (Howell et al 2010) and are briefly summarized below.

For in-field organs, the mean organ absorbed dose values from primary radiation were taken directly from the TPS. Absorbed dose was calculated in $2 \times 2 \times 2.5 \mathrm{~mm}^{3}$ voxels throughout the patient's body, and the mean organ absorbed dose from primary protons, $D_{T}$ (pri, IMRT), was determined using equation (1). For completely out-of-field organs, the mean organ absorbed dose values from stray radiation were determined from measurements made within an adult-sized male anthropomorphic phantom, with CT images of the phantom registered to those of the patients in the TPS and the treatment isocenters for the patient and the phantom anatomically registered. The phantom was irradiated using the patient's treatment plan, and the absorbed dose was measured using lithium fluoride TLDs at locations corresponding to the organs of interest. The mean organ absorbed dose from stray radiation, $D_{T}$ (str, IMRT), was calculated as an average of all TLD measurements within an organ and was normalized per therapeutic absorbed dose delivered to isocenter. For partially in-field organs, a sensitivity analysis was performed to determine if the DVH data were sufficiently accurate for calculating the mean organ dose. If the sensitivity analysis found that the accuracy of the out-of-field component of dose had a significant effect on the mean organ absorbed dose, $D_{T}$ (pri+str, IMRT) was calculated based on a volume-weighted 
technique, which combined the TPS estimate of the in-field component of dose with a measured out-of-field component of dose.

The $H_{T}$ value for each type of photon radiation was calculated using equation (2), with $\overline{w_{R}}$ of 1, following the recommendations of ICRP Publication 92.

\subsection{Risk of SMN incidence}

Lifetime absolute risk of SMN incidence, $I_{T}$, attributable to radiation exposures in PBT and IMRT treatments, was estimated according to

$$
I_{T}=\left(\frac{I_{T}}{H_{T}}\right) \cdot H_{T}
$$

where the risk coefficients $I_{T} / H_{T}$ (table 1 ) were taken directly from the age-, sex- and organspecific risk model in table 12D-1 of the BEIR VII Report (NRC 2006). This model from the BEIR VII Report assumes a risk-free latent period of 2 years for leukemia and 5 years for all other cancers. The $H_{T}$ values were determined according to sections 2.2 and 2.3 of this work. The risk coefficient for liver cancer was only applied to the $H_{T}$ value for nontumor liver.

\subsection{Risk of non-melanoma skin cancer incidence}

To calculate the organ dose in skin for IMRT, $D_{\text {skin }}$ (IMRT), and to predict the risk of nonmelanoma skin cancer (NMSC) incidence after IMRT and PBT, the methods described in sections $2.2-2.4$ were slightly modified. The values for $D_{\text {skin }}$ (IMRT) were estimated by taking a volume-weighted average of the in-field dose ( $8 \%$ of the skin volume) and out-offield dose ( $92 \%$ of skin volume). The out-of-field component of $D_{\text {skin }}$ (IMRT) was not measured in the previous study by Howell et al (2010). Therefore, for the out-of-field component of $D_{\text {skin }}$ (IMRT), we used data from a previous study by Howell et al (2006) for a 6 MV IMRT treatment, in terms of $D_{\text {skin }}$ (IMRT) per monitor unit (MU), and the number of MUs delivered in the liver study by Howell et al (2010). More specifically, we multiplied the out-of-field $D_{\text {skin }}$ (IMRT)/MU from Howell et al (2006) of $3.75 \times 10^{-6} \mathrm{~Gy} \mathrm{MU}^{-1}$ by the total MUs required to deliver the 60 Gy prescription dose from Howell et al (2010) of 38 $720 \mathrm{MU}$ to obtain $D_{\text {skin }}(\mathrm{IMRT}) . D_{\text {skin }}(\mathrm{PBT}), H_{\text {skin }}(\mathrm{IMRT})$ and $H_{\text {skin }}(\mathrm{PBT})$ were determined with the same methods as the other organs. Because a recommendation for the risk coefficient for NMSC, $I_{\text {skin }} / H_{\text {skin }}$, was not provided in the BEIR VII Report, we applied the risk coefficient recommended by the ICRP in Publication 60 (ICRP 1991). To correct for age at exposure, we multiplied $I_{\text {skin }} / H_{\text {skin }}$ by an age-correction factor of 0.45 . The final value of $I_{\text {skin }} / H_{\text {skin }}$ is listed in table 1 .

\section{Results}

\subsection{Organ doses}

Table 2 lists the mean equivalent doses in organs and tissues from primary and stray radiation for PBT and IMRT. For organs that were in or near the primary field, the primary field dominated the mean organ dose. For organs that were far away from the primary field, however, the stray radiation dominated the mean organ dose. Figure 1 plots DVHs for primary radiation. Figure 2 shows the distributions of $D_{v}$ in the axial, sagittal and coronal planes through isocenter from primary radiation of PBT and IMRT. The superior normal tissue sparing of primary radiation doses from PBT relative to IMRT is evident in these figures, particularly for the esophagus, stomach, left kidney and non-tumor liver. In the IMRT plan, $63 \%$ of the non-tumor liver received at least $10 \mathrm{~Gy}$, while only $29 \%$ of the non- 
tumor liver received at least $10 \mathrm{~Gy}$ in the PBT plan. However, the DVHs for the non-tumor liver were similar above $30 \mathrm{~Gy}$, with the PBT plan having slightly more non-tumor liver in the high-dose region. Importantly, the mean dose in the non-tumor liver was less with PBT.

Because the absorbed dose values from primary radiation for PBT and IMRT were based on the absorbed doses calculated by the TPS, the uncertainties in these values were assumed to be less than 3\% in-field. The statistical uncertainties in the mean equivalent dose in each organ or tissue from stray radiation for PBT were less than $3 \%$ for the Monte Carlo calculations and were reported at the $68 \%$ confidence interval. The measurement uncertainty was less than 3\% for each TLD measurement, so the mean equivalent dose from stray radiation for IMRT was less than 3\% for each organ or tissue. Together, the uncertainties in the primary and stray radiation mean equivalent dose values in each organ or tissue were less than $3 \%$ for PBT and IMRT.

\subsection{Risk of SMN incidence}

Figure 3 plots the predicted lifetime risks of SMN incidence by cancer site after PBT and IMRT. For each treatment, the risks take into account both primary and stray radiation. The total risks of SMN incidence, summed over all cancer sites, were $11.4 \%$ and $19.2 \%$ after PBT and IMRT, respectively. For PBT, approximately one-fourth of the predicted risk was from stray radiation.

\section{Discussion}

In this comparison of the predicted risk of SMN incidence after PBT or IMRT for a 59-yearold male patient with HCC, we found that PBT reduced the risk by approximately $40 \%$ relative to IMRT (from 19.2 to $11.4 \%$ lifetime risk). Additionally, we found that PBT spared the non-tumor liver and other normal tissues more than IMRT, which may lead to reduced incidences of RILD, other toxicities and other normal tissue complications. Reducing dose to the non-tumor liver also introduces the possibility of escalating dose to the target volume.

Liver cancer is a deadly disease, and novel and improved radiation treatment strategies are urgently needed to reduce normal tissue toxicities. Our data revealed that using PBT almost halved the predicted risk of SMN incidence in a long-term survivor of liver cancer. Reducing SMN risk is becoming more important as the long-term prognosis improves for liver cancer patients who are candidates for radiotherapy. For example, in HCC patients who received PBT as a bridge to liver transplantation, 5-year survival rates have reached 70\% (Llovet 2005). In fact, in a group of HCC patients who had solitary tumors and minimal impairment of hepatic function due to cirrhosis, the reported 5-year survival rate achieved by PBT was greater than $50 \%$, rivaling the outcomes reported for similar cases following surgical resection (Chiba et al 2005). These promising results suggest that radiotherapy can be employed with curative intent in the treatment of HCC.

While the therapeutic fields dominated the risk after both PBT and IMRT, the risk from stray radiation was substantial, and reasonable measures should be taken to reduce the normal tissue doses from stray radiation in PBT (Taddei et al 2008, 2009d, Brenner et al 2009) and in IMRT (Kry et al 2010, Chofor et al 2010). Quantifying and reducing stray radiation exposure appear to be manageable tasks in developing PBT and IMRT treatments for liver cancer.

Sparing the non-tumor liver from radiation dose is of particular importance for reducing the probability of RILD, which is often fatal; patients with underlying cirrhosis and hepatic dysfunction are at particularly high risk of death due to RILD. The mean equivalent dose in 
the non-tumor liver was $29 \%$ less for PBT than for IMRT, which suggests the possibility of reducing the risk of RILD.

Our findings are in good agreement with previous dosimetric studies. Wang et al (2008) performed a dosimetric comparison between photon radiotherapy and proton beam radiotherapy for nine patients with intrahepatic malignancies. From their TPS-based dosimetric comparisons, they concluded that protons were superior to photons in conforming the dose to the target and reducing dose to critical structures. However, they neglected stray radiation, which is especially important outside the treatment volume.

One limitation of our study was that it was theoretical. That is, we predicted radiation exposures and risks for a single patient rather than following up a cohort of patients for many years. We relied on risk models from the literature that were derived from data from a general population, mainly atomic bomb survivors, and the application of these risk models to a cancer patient introduced uncertainty in the risk predictions that is difficult to quantify (NRC 2006,Fontenot et al 2009,Newhauser et al 2009). Confirmatory patient follow-up studies are needed but may take a decade or more due to the latency time of some SMNs.

In summary, the findings of this study suggest that using PBT rather than IMRT reduced the predicted lifetime risk of SMN incidence in a patient with HCC.

\section{Acknowledgments}

The authors would like to acknowledge John Carr and Jaques Bluett for their assistance in treatment planning and Daniel Jackson for assistance with the computational infrastructure. We thank Dr Jonas Fontenot for helpful scientific discussions. We are indebted to Kathryn B Carnes and John $\mathrm{H}$ McCool for their assistance in the preparation of this manuscript. This work was supported in part by the National Cancer Institute (awards 1R01CA131463-01A1 and 5K01CA125204-04), by the Fogarty International Center (award K01TW008409) and by Northern Illinois University through a subcontract of Department of Defense (award W81XWH-08-1-0205). The content is solely the responsibility of the authors and does not necessarily represent the official views of the sponsors.

\section{References}

Agosteo S, Birattari C, Caravaggio M, Silari M, Tosi G. Secondary neutron and photon dose in proton therapy. Radiother. Oncol 1998;48:293-305. [PubMed: 9925249]

Altekruse SF, McGlynn KA, Reichman ME. Hepatocellular carcinoma incidence, mortality and survival trends in the United States from 1975 to 2005. J. Clin. Oncol 2009;27:1485-1491. [PubMed: 19224838]

Brenner DJ, Elliston CD, Hall EJ, Paganetti H. Reduction of the secondary neutron dose in passively scattered proton radiotherapy, using an optimized pre-collimator/collimator. Phys. Med. Biol 2009;54:6065-6078. [PubMed: 19779218]

Chiba T, et al. Proton beam therapy for hepatocellular carcinoma: a retrospective review of 162 patients. Clin. Cancer Res 2005;11:3799-3805. [PubMed: 15897579]

Chofor N, Harder D, Ruhmann A, Willborn KC, Wiezorek T, Poppe B. Experimental study on photonbeam peripheral doses, their components and some possibilities for their reduction. Phys. Med. Biol 2010;55:4011-4027. [PubMed: 20577041]

Dawson LA. Protons or photons for hepatocellular carcinoma? Let's move forward together. Int. J. Radiat. Oncol. Biol. Phys 2009;74:661-663. [PubMed: 19480966]

Fontenot JD, Lee AK, Newhauser WD. Risk of secondary malignant neoplasms from proton therapy and intensity-modulated x-ray therapy for early-stage prostate cancer. Int. J. Radiat. Oncol. Biol. Phys 2009;74:616-622. [PubMed: 19427561]

Fontenot JD, Taddei P, Zheng Y, Mirkovic D, Jordan T, Newhauser WD. Equivalent dose and effective dose from stray radiation during passively scattered proton radiotherapy for prostate cancer. Phys. Med. Biol 2008;53:1677-1688. [PubMed: 18367796] 
Garcia, M.; Jemal, A.; Ward, EM.; Center, MM.; Hao, Y.; Siegel, RL.; Thun, MJ. Global Cancer Facts \& Figures 2007. Atlanta, GA: American Cancer Society; 2007.

Horner, MJ., et al., editors. SEER Cancer Statistics Review 1975-2006. Bethesda, MD: National Cancer Institute; 2008.

Howell RM, Hertel NE, Wang Z, Hutchinson J, Fullerton GD. Calculation of effective dose from measurements of secondary neutron spectra and scattered photon dose from dynamic MLC IMRT for 6 MV, 15 MV and 18 MV beam energies. Med. Phys 2006;33:360-368. [PubMed: 16532941]

Howell RM, Scarboro SB, Taddei PJ, Krishnan S, Kry SF, Newhauser WD. Methodology for determining doses to in-field, out-of-field and partially in-field organs for late effects studies in photon radiotherapy. Phys. Med. Biol 2010;55:7009-7023. [PubMed: 21076193]

ICRP. Recommendations of the International Commission on Radiological Protection. Publication 60. Ann. ICRP 1991;21

ICRP. Relative Biological Effectiveness (RBE), Quality Factor $(Q)$, and Radiation Weighting Factor $\left(w_{R}\right)$. Publication 92. Ann. ICRP 2003;33

Krishnan S, Dawson LA, Seong J, Akine Y, Beddar S, Briere TM, Crane CH, Mornex F. Radiotherapy for hepatocellular carcinoma: an overview. Ann. Surg. Oncol 2008;15:1015-1024. [PubMed: 18236114]

Kry SF, Vassiliev ON, Mohan R. Out-of-field photon dose following removal of the flattening filter from a medical accelerator. Phys. Med. Biol 2010;55:2155-2166. [PubMed: 20305334]

Llovet JM. Updated treatment approach to hepatocellular carcinoma. J. Gastroenterol 2005;40:225235. [PubMed: 15830281]

NCRP. NCRP Report 116. Bethesda, MD: National Council on Radiation Protection and Measurements; 1993. Limitation of exposure to ionizing radiation.

Newhauser W, Fontenot J, Zheng Y, Polf J, Titt U, Koch N, Zhang X, Mohan R. Monte Carlo simulations for configuring and testing an analytical proton dose-calculation algorithm. Phys. Med. Biol 2007a;52:4569-4584. [PubMed: 17634651]

Newhauser W, et al. A Monte-Carlo based dose engine for proton radiotherapy treatment planning. Med. Phys 2007b;34:2406.

Newhauser WD, et al. The risk of developing a second cancer after receiving craniospinal proton irradiation. Phys. Med. Biol 2009;54:2277-2291. [PubMed: 19305036]

Newhauser WD, Giebeler A, Langen KM, Mirkovic D, Mohan R. Can megavoltage computed tomography reduce proton range uncertainties in treatment plans for patients with large metal implants? Phys. Med. Biol 2008a;53:2327-2344. [PubMed: 18421122]

Newhauser WD, Zheng Y, Taddei PJ, Mirkovic D, Fontenot JD, Giebeler A, Zhang R, Titt U, Mohan R. Monte Carlo proton radiation therapy planning calculations. T. Am. Nucl. Soc 2008b;99:63-64.

NRC (National Research Council). Health Risks from Exposure to Low Levels of Ionizing Radiation: BEIR VII-Phase 2. Washington, DC: National Academies Press; 2006.

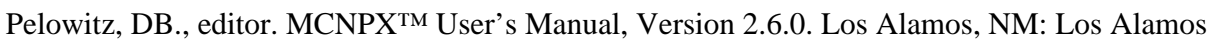
National Laboratory; 2008.

Perz JF, Armstrong GL, Farrington LA, Hutin YJ, Bell BP. The contributions of hepatitis B virus and hepatitis $\mathrm{C}$ virus infections to cirrhosis and primary liver cancer worldwide. J. Hepatol 2006;45:529-538. [PubMed: 16879891]

Taddei PJ, Fontenot JD, Zheng Y, Mirkovic D, Lee AK, Titt U, Newhauser WD. Reducing stray radiation dose to patients receiving passively scattered proton radiotherapy for prostate cancer. Phys. Med. Biol 2008;53:2131-2147. [PubMed: 18369278]

Taddei, PJ.; Krishnan, S.; Mirkovic, D.; Yepes, P.; Newhauser, WD. Effective dose from stray radiation for a patient receiving proton therapy for liver cancer; AIP Conf. Proc; 2009a. p. 445-449.

Taddei PJ, Mirkovic D, Fontenot JD, Giebeler A, Zheng Y, Kornguth D, Mohan R, Newhauser WD. Stray radiation dose and second cancer risk for a pediatric patient receiving craniospinal irradiation with proton beams. Phys. Med. Biol 2009b;54:2259-2275. [PubMed: 19305045]

Taddei PJ, Mirkovic D, Fontenot JD, Giebeler A, Zheng Y, Kornguth D, Mohan R, Newhauser WD. Stray radiation dose and second cancer risk for a pediatric patient receiving craniospinal irradiation with proton beams. Phys. Med. Biol 2009c;54:2259-2275. [PubMed: 19305045] 
Taddei PJ, Mirkovic D, Fontenot JD, Giebeler A, Zheng Y, Titt U, Woo S, Newhauser WD. Reducing stray radiation dose for a pediatric patient receiving proton craniospinal irradiation. Nucl. Technol 2009d;168:108-112. [PubMed: 20865143]

Wang X, Krishnan S, Zhang X, Dong L, Briere T, Crane CH, Martel M, Gillin M, Mohan R, Beddar S. Proton radiotherapy for liver tumors: dosimetric advantages over photon plans. Med. Dosim 2008;33:259-267. [PubMed: 18973852]

Zheng Y, Fontenot J, Taddei P, Mirkovic D, Newhauser W. Monte Carlo simulations of neutron spectral fluence, radiation weighting factor and ambient dose equivalent for a passively scattered proton therapy unit. Phys. Med. Biol 2008;53:197-201.

Zurlo A, Lomax A, Hoess A, Bortfeld T, Russo M, Goitein G, Valentini V, Marucci L, Capparella R, Loasses A. The role of proton therapy in the treatment of large irradiation volumes: a comparative planning study of pancreatic and biliary tumors. Int. J. Radiat. Oncol. Biol. Phys 2000;48:277288. [PubMed: 10924999] 


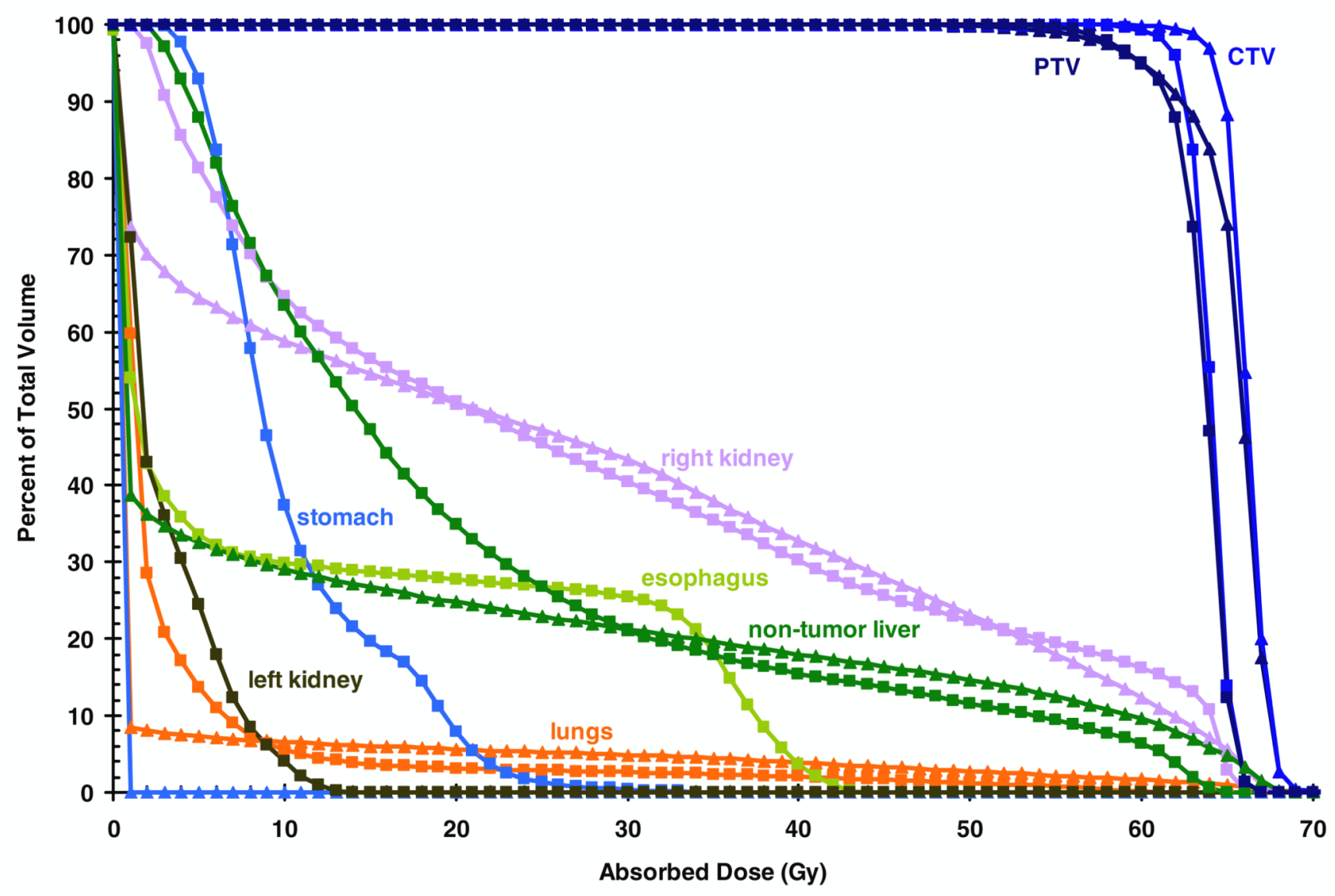

Figure 1.

DVHs from the primary fields for in-field or partially in-field structures, normalized so that 95\% of the PTV receives the prescribed dose of $60 \mathrm{~Gy}$. DVHs for the CTV, PTV of the IMRT plan, right kidney, esophagus, non-tumor liver, stomach, left kidney and lungs are shown for the PBT (triangles) and IMRT (squares) plans. 


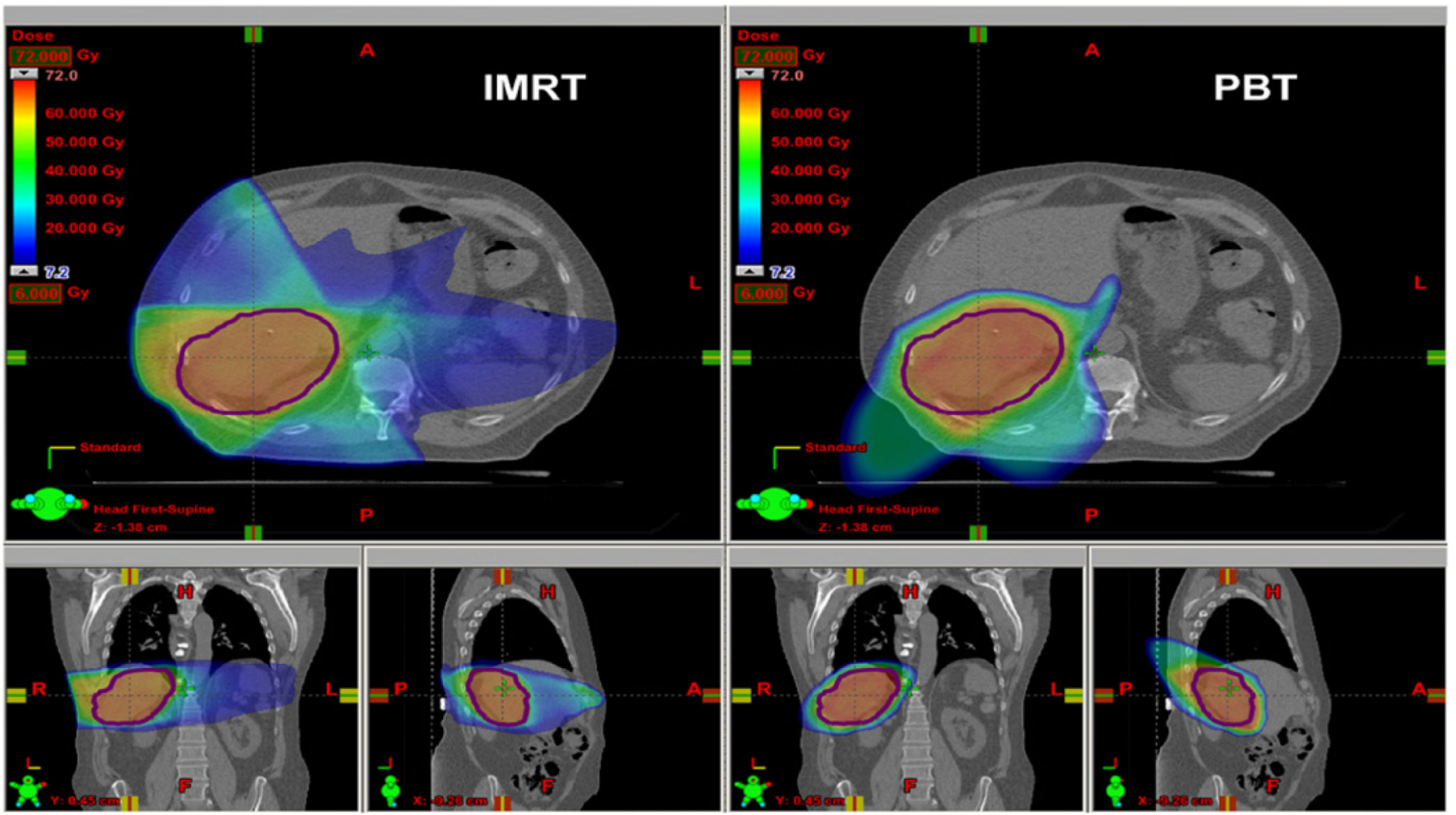

Figure 2.

Predicted dose distributions from the primary fields for IMRT (left) and PBT (right) in the axial (top), coronal (lower left) and sagittal (lower right) planes through isocenter. The contour marked in purple is for the CTV. 


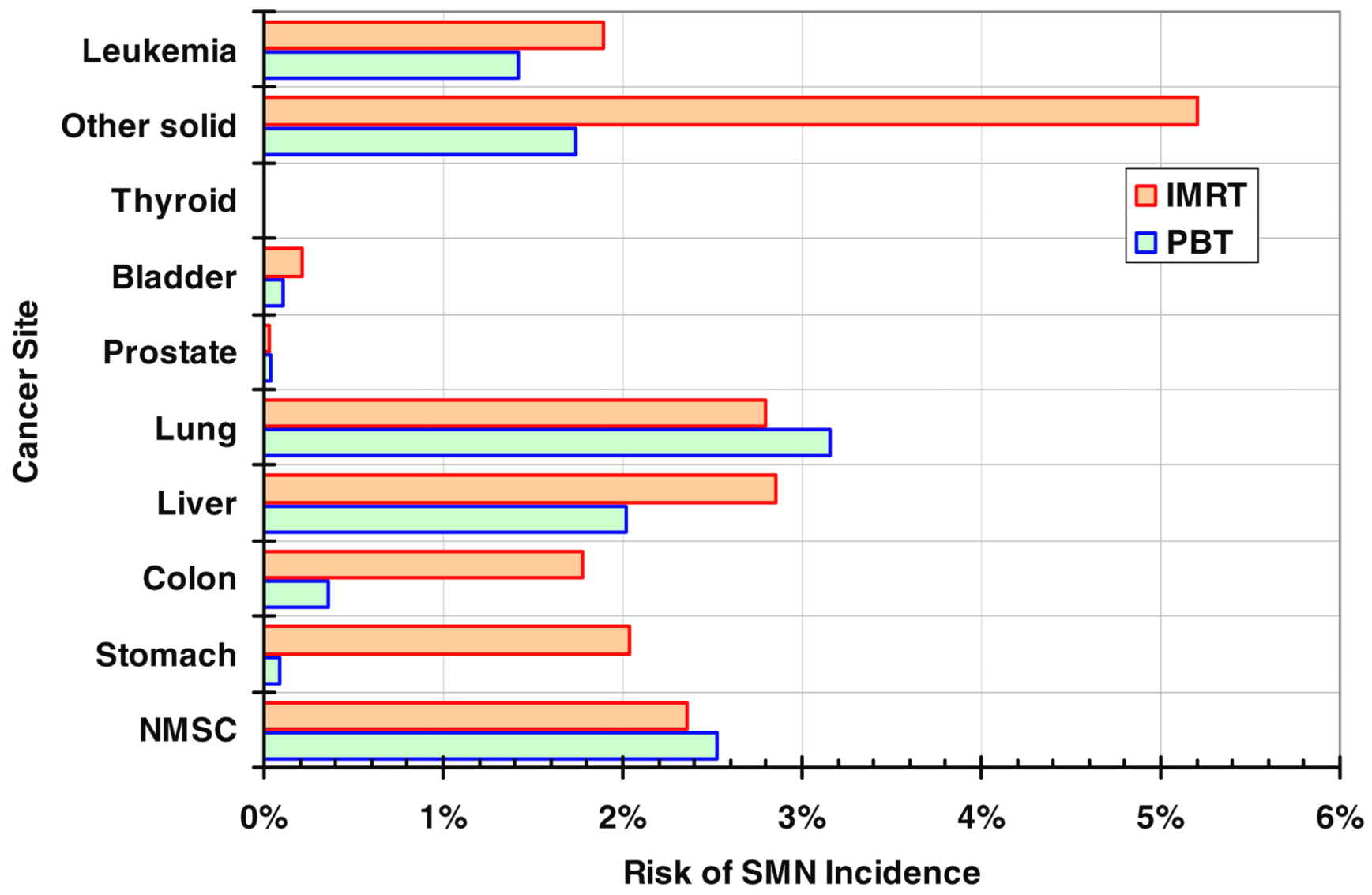

Figure 3.

Site-specific predicted lifetime risks of SMN incidence after PBT and IMRT. Other solid SMN risks are based on the mean dose in the non-tumor body tissue. Leukemia risks are based on the mean dose in the red bone marrow. 


\section{Table 1}

Risk coefficients for SMN incidence, $I_{T} / H_{T}$, for 60 -year-old males from table 12D-1 in the BEIR VII report (NRC 2006), except for $I_{\text {skin }} / H_{\text {skin }}$, which was from ICRP Publication 60 (ICRP 1991), listed in terms of absolute risk (in percent) per equivalent dose.

\begin{tabular}{ll}
\hline Cancer site & $\boldsymbol{I}_{T} / H_{T}(\% / S v)$ \\
\hline Stomach & 0.20 \\
Colon & 0.94 \\
Liver & 0.14 \\
Lung & 0.89 \\
Prostate & 0.26 \\
Bladder & 0.66 \\
Other & 0.98 \\
Thyroid & 0.003 \\
Leukemia & 0.82 \\
Skin & 2.2 \\
\hline
\end{tabular}




\section{Table 2}

Mean organ equivalent dose PBT, including primary proton radiation, stray (external and internal) neutron radiation (from Taddei et al (2009a)) and a sum of both primary and stray radiation. For IMRT, the combined dose from both primary and stray radiation (from Howell et al (2010) and Howell et al (2006)) is listed. The statistical uncertainty of each value is less than $3 \%$.

\begin{tabular}{lrrrr}
\hline Organ or tissue & $\begin{array}{r}\text { PBT } \\
\text { primary (Sv) }\end{array}$ & $\begin{array}{r}\text { PBT } \\
\text { stray (Sv) }\end{array}$ & $\begin{array}{r}\text { PBT } \\
\text { total (Sv) }\end{array}$ & $\begin{array}{r}\text { IMRT } \\
\text { total (Sv) }\end{array}$ \\
\hline CTV & 66.11 & 1.89 & 68.00 & 63.99 \\
PTV & 65.29 & 1.82 & 67.11 & 63.52 \\
Whole liver & 23.64 & 1.03 & 24.67 & 28.62 \\
Non-tumor liver & 13.61 & 0.84 & 14.45 & 20.40 \\
Right kidney & 25.66 & 1.36 & 27.02 & 27.00 \\
Left kidney & 0.00 & 0.38 & 0.38 & 3.59 \\
Stomach & 0.01 & 0.45 & 0.46 & 10.20 \\
Colon & 0.05 & 0.33 & 0.38 & 1.89 \\
Lungs & 2.97 & 0.57 & 3.54 & 3.14 \\
Prostate & 0.00 & 0.16 & 0.16 & 0.13 \\
Bladder & 0.00 & 0.16 & 0.16 & 0.32 \\
Thyroid & 0.00 & 0.26 & 0.26 & 0.46 \\
Red bone marrow & 1.34 & 0.39 & 1.73 & 2.31 \\
Skin & 0.77 & 0.36 & 1.13 & 1.06 \\
Esophagus & 0.74 & 0.61 & 1.35 & 11.20 \\
Whole body & 1.85 & 0.36 & 2.21 & 6.17 \\
Non-tumor body tissue & 1.41 & 0.36 & 1.77 & 5.31 \\
\hline
\end{tabular}

